\title{
Excitation spectrum for ultrafast photogeneration of charged solitons in polyacetylene
}

\author{
Paulo B. Miranda,* Daniel Moses, and Alan J. Heeger ${ }^{\dagger, *}$ \\ Institute for Polymers and Organic Solids, University of California at Santa Barbara, Santa Barbara, California 93106 \\ Y. W. Park \\ School of Physics, Seoul National University, Seoul 151747, South Korea
}

(Received 22 April 2002; published 10 September 2002)

\begin{abstract}
Ultrafast photoinduced absorption by infrared-active vibrational modes is used to detect charged solitons in oriented trans-polyacetylene. Soliton pairs are photogenerated within $\sim 250 \mathrm{fs}$ with quantum efficiencies $\left(\phi_{\mathrm{ch}}\right)$ approaching unity. The excitation spectrum of $\phi_{\mathrm{ch}}$ shows an onset at $\sim 1.0 \mathrm{eV}$ with a weak photon energy dependence up to $4.7 \mathrm{eV}$. The results are consistent with the ultrafast soliton formation predicted by $\mathrm{Su}$ and Schrieffer and with the Su-Scrieffer-Heeger threshold of $2 E_{g} / \pi$ for soliton pair production. The recombination dynamics of charged solitons is very fast (initial decay $<1 \mathrm{ps}$ ) with a modest dependence on the pump photon energy.
\end{abstract}

DOI: 10.1103/PhysRevB.66.125202

PACS number(s): 78.47.+p, 78.66.Qn, 73.21.-b, 73.61.Ph

\section{INTRODUCTION}

The discovery of electrical conductivity in doped polyacetylene ${ }^{1}$ initiated the field of semiconducting and metallic polymers. Motivated in part by their potential for use in applications, this novel class of materials, which combine the optical and electronic properties of semiconductors and metals with the mechanical properties and processability of polymers, continues to be the focus of an active field of research. $^{2}$

Polyacetylene $(\mathrm{CH})_{x}$ is the simplest semiconducting (conjugated) polymer. The theoretical description of the degenerate ground state, the electronic structure and the soliton excitations of trans- $(\mathrm{CH})_{x}$ has been generalized to the broader class of semiconducting polymers in which the ground state degeneracy has been lifted. The successful model of $\mathrm{Su}$, Schrieffer, and Heeger (SSH) (Ref. 3) treats the polymer chain as a tight-binding one-dimensional semiconductor in the one-electron approximation and explicitly includes the electron-phonon interaction, but neglects electron-electron (el-el) interactions, assuming they are relatively weak due to screening. The SSH model predicts that the lowest energy fundamental excitations in polyacetylene are solitons, selflocalized by the electron-phonon interaction. The solitons in trans- $(\mathrm{CH})_{x}$ have a reversed spin-charge relationship; neutral solitons $\left(S^{0}\right)$ have spin $\frac{1}{2}$, and charged solitons $\left(S^{+}, S^{-}\right)$ have spin 0 . The minimum energy required for creation of a soliton pair was calculated to be $2 E_{g} / \pi$ within the $\mathrm{SSH}$ model, where $E_{g}$ is the $\pi$ - $\pi^{*}$ energy gap. Solitons in polyacetylene have been studied experimentally by several techniques. $^{3}$

Calculations by $\mathrm{Su}$ and $\mathrm{Schrieffer}^{4}$ described the process of charged soliton pair formation subsequent to photoexcitation via the $\pi-\pi^{*}$ interband transition in trans $-(\mathrm{CH})_{x}$ with a photon energy $(h \nu)$ equal to $E_{g}$. They showed that within $\sim 100 \mathrm{fs}$, the lattice around a photoexcited electron-hole pair relaxes to form a well separated $S^{+}-S^{-}$pair. Other calculations have shown that this picture remains correct when weak electron-electron interactions are included in the model. ${ }^{5}$ The predicted ultrafast photogeneration of solitons in trans- $(\mathrm{CH})_{x}$ has been confirmed with time-resolved spectroscopy. ${ }^{6-9}$ These early ultrafast experiments used photoexcitation energies above the energy gap $\left(h \nu \geqslant E_{g}\right)$. However, direct photogeneration of soliton pairs can occur even for $2 E_{g} / \pi<h \nu<E_{g}$, enabled by nonlinear zero-point motion of the lattice. ${ }^{10}$ The absorption coefficient for direct soliton photoexcitation was predicted to be small near the threshold $2 E_{g} / \pi$, and to increase exponentially as the photon energy approaches $E_{g} .{ }^{11}$ If this is the dominant absorption process in the trans $-(\mathrm{CH})_{x}$ samples below the energy gap, one would expect that the quantum efficiency for charged soliton pair photogeneration $\left(\phi_{\mathrm{ch}}\right)$ to be unity for $2 E_{g} / \pi<h \nu<E_{g}$ and, if the charged soliton pair is the lowest energy photoexcitation, well above $E_{g}$.

This issue has been addressed experimentally by measurements of steady-state photoinduced absorption (PIA) (Refs. 12 and 13) and photoconductivity, ${ }^{14}$ yielding the excitation spectrum for charged soliton generation. The excitation spectrum drops exponentially from $E_{g}$ towards an onset at $\sim 1.0$ $\mathrm{eV}$, and $\phi_{\mathrm{ch}}$ was estimated to be only about $1 \%$ for energies above $E_{g}$. These results have been interpreted both in support of (since the onset is consistent with $\left.2 E_{g} / \pi\right)^{12}$ and against the SSH model (since $\phi_{\mathrm{ch}} \sim 1 \%$ at $2.0 \mathrm{eV}$ and is reduced to $0.1 \%$ at $1.4 \mathrm{eV}) .{ }^{13}$ However, steady-state measurements do not probe the early time intrinsic photoexcitations, but only the long-lived solitons that are usually associated with traps due to defects and impurities. 5,9 Measurements with femtosecond time resolution are needed to obtain the excitation spectrum for intrinsic photogeneration of soliton pairs.

Here we use ultrafast photoinduced absorption by infrared-active vibrational (IRAV) modes, ${ }^{15,16}$ an all-optical technique with subpicosecond time resolution carried out in zero applied electric field, to investigate soliton photogeneration and dynamics in oriented trans-polyacetylene. The IRAV absorption results from Raman-active vibrational modes that become infrared-active when the local symmetry is broken by self-localization of charges with associated changes in the bond lengths (e.g., soliton formation). The IRAV modes have a one-to-one correspondence with the 
strongest modes observed in resonant Raman scattering and have an unusually high infrared absorption crosssection $\left(\sigma_{\text {IRAV }} \sim 10^{-16} \mathrm{~cm}^{2}\right.$, comparable to electronic transitions). ${ }^{3,9,17}$ The intensities of the IRAV modes are a well-known probe of photo-induced or doping-induced charged excitations (solitons, polarons, and bipolarons) in conjugated polymers. ${ }^{3,18,19}$ Thus, ultrafast photoinduced IRAV absorption can probe the early time photogeneration and dynamics of charged solitons in polyacetylene, but without being sensitive to neutral solitons.

\section{EXPERIMENTAL DETAILS}

Free-standing oriented film of trans-polyacetylene (Shirakawa synthesis ${ }^{20}$ ), with draw ratio of 4 and $8 \mu \mathrm{m}$ thick, were used. When doped with $\mathrm{FeCl}_{3}$, these high quality samples have electrical conductivities of $\sim 25000 \mathrm{~S} / \mathrm{cm}$ at room temperature. ${ }^{21}$ The trans- $(\mathrm{CH})_{x}$ film was mounted in a cryostat with two $6 \mathrm{~mm}$ thick $\mathrm{BaF}_{2}$ windows. The sample handling was done inside a glovebox under $\mathrm{N}_{2}$ atmosphere. All experiments were performed with the sample at room temperature $(298 \mathrm{~K})$ in a vacuum better than $10^{-5}$ mbar. A few measurements were done at $\sim 80 \mathrm{~K}$; the results were indistinguishable from the data obtained at room temperature. The pulsed laser facility consisted of an amplified Ti:sapphire system (wavelength $795 \mathrm{~nm}, 1 \mathrm{~mJ} /$ pulse, $\sim 100 \mathrm{fs}$ pulse duration, $1 \mathrm{kHz}$ repetition rate) pumping two optical parametric amplifiers (OPA's). The first OPA was used to pump the samples and generated pulses tunable from 1500 to $300 \mathrm{~nm}$ by nonlinear mixing of pump, signal, and idler beams. Additional pump wavelengths were obtained by second- and third-harmonic generation of the fundamental wavelength. The second OPA was equipped with a difference-frequency generation stage and generated the infrared (IR) probe beam, tunable from 3 to $10 \mu \mathrm{m}$ (1000 to $3300 \mathrm{~cm}^{-1}$ ). The pump and probe pulses overlapped on the sample and the delay between them was controlled with a variable delay line. The beam sizes (1/e intensity radius) at the sample position were $70 \mu \mathrm{m}$ for the probe beam, and varied from 130 to $400 \mu \mathrm{m}$ for the pump beam, depending on the wavelength. Polarizations were linear, with the IR polarized along the stretch direction of the sample. The pump could be polarized either parallel or perpendicular to the stretch direction (since the samples were optically thick to the pump beam for either polarization, no difference was observed). The pump beam was modulated with a mechanical chopper, and the differential changes in transmission $\left[\Delta T / T=\left(T_{\text {on }}-T_{\text {off }}\right) / T_{\text {off }}\right]$ of the probe induced by the pump beam were measured as a function of the pump-probe delay $(t)$ with a lock-in amplifier. The smallest detectable signal was $\Delta T / T=10^{-3}$, and the typical pump fluence on the sample was $<300 \mu \mathrm{J} / \mathrm{cm}^{2}$, resulting in a maximum excitation density at the absorption peak of the order of 5 $\times 10^{20} \mathrm{~cm}^{-3}$. Each measurement of $\Delta T / T(t)$ was fit to a convolution of multiple exponential decays and a Gaussian representing the temporal resolution of the experiment (typically about $250 \mathrm{fs})$. From the fit, we extracted the initial differential transmission $\Delta T / T(0)$, which is related to the quantum efficiency for charge pair generation $\left(\phi_{\mathrm{ch}}\right)$ by

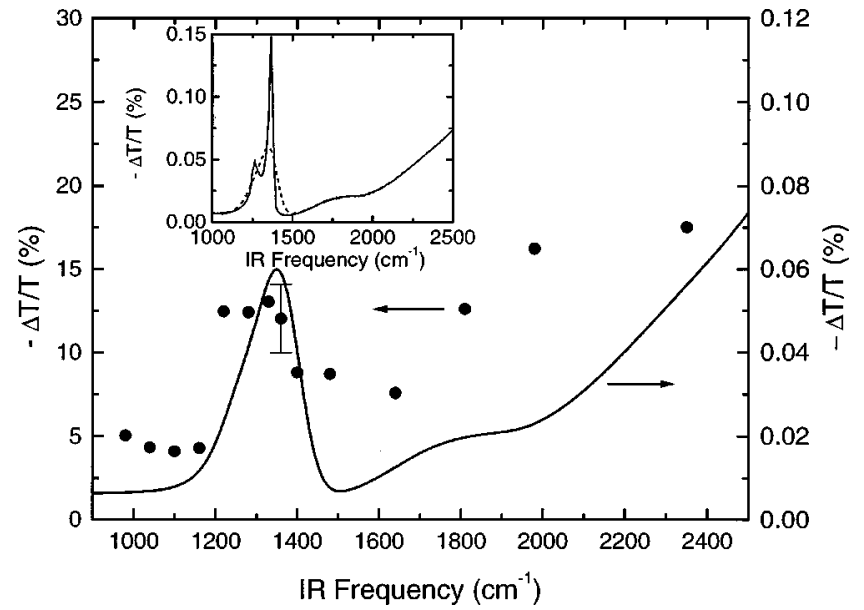

FIG. 1. Photoinduced IRAV spectrum for oriented transpolyacetylene. Solid circles are the ultrafast measurements extrapolated to $t=0[-\Delta T / T(0)]$. The inset shows the steady-state IRAV spectrum for unoriented trans-polyacetylene from Ref. 22 (solid line) and its convolution with a Gaussian with $100 \mathrm{~cm}^{-1}$ FWHM, representing the ultrafast IR laser bandwidth (dashed line). For comparison, this convoluted spectrum is plotted as a solid line together with the ultrafast measurements in the main panel. The pump wavelength and sample temperature were $662 \mathrm{~nm}$ and $298 \mathrm{~K}$ for the ultrafast measurements, and $514 \mathrm{~nm}$ and $10 \mathrm{~K}$ for the steady-state measurements.

$\Delta T / T(0)=2 \phi_{\mathrm{ch}} \sigma F$, where $F$ is the photon flux absorbed by the sample (photons $/ \mathrm{cm}^{2}$ ) and $\sigma$ is the cross section for IRAV absorption at the probe wavelength.

\section{RESULTS AND DISCUSSION}

Figure 1 compares the spectra of the IRAV modes obtained with steady-state excitation ${ }^{22}$ and with the ultrafast instrumentation. The inset shows the steady-state data from Ref. 22 (solid line) and its convolution with a Gaussian of $100 \mathrm{~cm}^{-1}$ full width at half maximum (FWHM), representing the ultrafast IR laser bandwidth (dashed line). This convoluted spectrum is then plotted as a solid line in Fig. 1 for a comparison with the spectrum measured with the ultrafast laser (solid points). These points are the values of $-\Delta T / T(0)$ extracted from curve fitting to the results of a $-\Delta T / T(t)$ measurement at each IR frequency. The steadystate and ultrafast spectra are in good agreement, with all the spectral features present in both. The ultrafast photoinduced IRAV modes demonstrate unequivocally that charged solitons are produced in less than $250 \mathrm{fs}$, consistent with the early predictions of $\mathrm{Su}$ and Schrieffer. ${ }^{4}$ Although also consistent with previous ultrafast measurements, ${ }^{6,7}$ the IRAV data provide the first evidence that the bond length distortions which form the soliton pair are generated in less than $250 \mathrm{fs}$.

Figure 2 shows two measurements of the IRAV photoinduced absorption (signal vs time) for excitation at two different photon energies, with their corresponding best fit lines. The curves were normalized for easy comparison of the charged soliton recombination dynamics. It can be seen that the dynamics of charged solitons is very fast, $<1 \mathrm{ps}$ for 


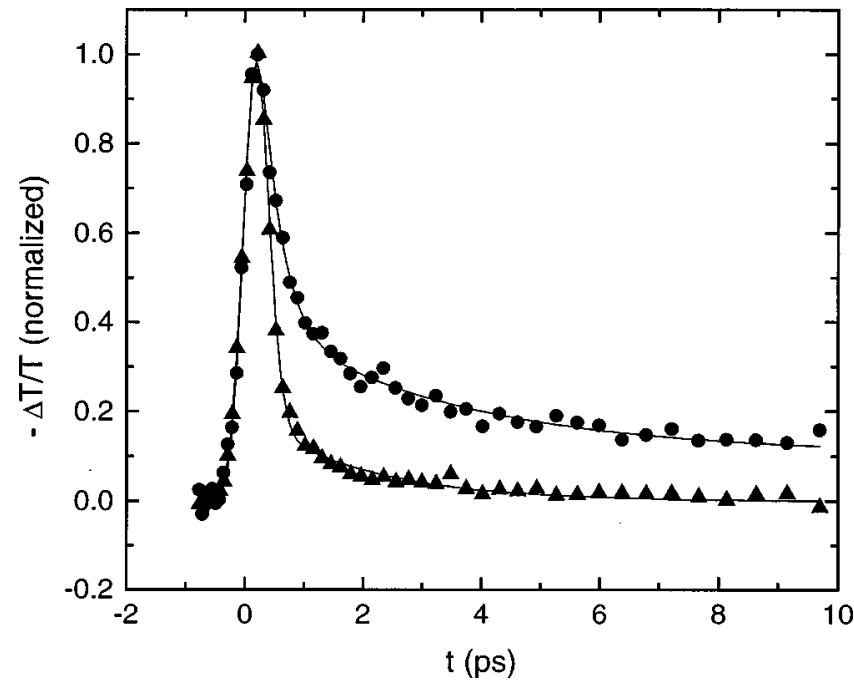

FIG. 2. Dynamics of the IRAV photoinduced absorption in oriented trans-polyacetylene. The pump photon energy was $1.87 \mathrm{eV}$ (triangles) and $4.7 \mathrm{eV}$ (circles). The IR probe was at $1360 \mathrm{~cm}^{-1}$. The solid lines are fits to multiple exponential decays, convoluted with a Gaussian representing our time resolution (see text for details). The curves were normalized for easy comparison.

excitation near the absorption maximum (triangles). This has been previously observed by other ultrafast studies. ${ }^{6,9}$ Kivelson and $\mathrm{Wu}^{5}$ explained the fast recombination as resulting from charge recombination as charged solitons separate after the optical excitation, leading to the formation of neutral soliton pairs which decay into the ground state. In our experiments, where the IRAV modes (at $\sim 0.17 \mathrm{eV}$ ) probe only charged solitons, the recombination dynamics appears even faster than previously reported. ${ }^{6,7,9}$

To our knowledge, all ultrafast measurements to date have used excitation wavelengths near the absorption maximum. Here we vary the excitation photon energy over a wide range. We found that the recombination dynamics depends on the excitation wavelength, as shown in Fig. 2. For 1.87 $\mathrm{eV}$ excitation, the dynamics can be fitted with two exponential decays of lifetimes $0.099 \pm 0.024 \mathrm{ps}$ and $1.48 \pm 0.21 \mathrm{ps}$, with amplitudes $4.67 \pm 1.08$ and $0.21 \pm 0.03$, respectively. Pumping at $4.67 \mathrm{eV}$ leads to slower dynamics and a longlived tail, so the curve was fitted with three exponential decays. Their lifetimes are $0.33 \pm 0.04 \mathrm{ps}, 3.31 \pm 0.37 \mathrm{ps}$, and $189 \pm 36 \mathrm{ps}$, with amplitudes $1.31 \pm 0.09,0.31 \pm 0.03$, and $0.09 \pm 0.01$, respectively. Both the lifetimes and the fraction of longer-lived charged excitations increase for excitation at higher photon energies. Other measurements (not shown) indicate that for photon energies $0.86 \leqslant h \nu \leqslant 3.0 \mathrm{eV}$, the dynamics is very similar to that with $1.87 \mathrm{eV}$ excitation. For $h \nu \geqslant 3.0 \mathrm{eV}$, the recombination dynamics evolves toward the data shown for pumping at $4.67 \mathrm{eV}$.

We do not propose a specific explanation for this change in dynamics with excitation photon energy. However, because the recombination process involves inter-conversion from well separated (relaxed) ${ }^{23}$ charged to neutral soliton pairs (as suggested by Kivelson and $\mathrm{Wu}^{5}$ ), highly excited charge pairs might take considerably longer to attain a relaxed state, ${ }^{24}$ where efficient recombination can occur. In ad-

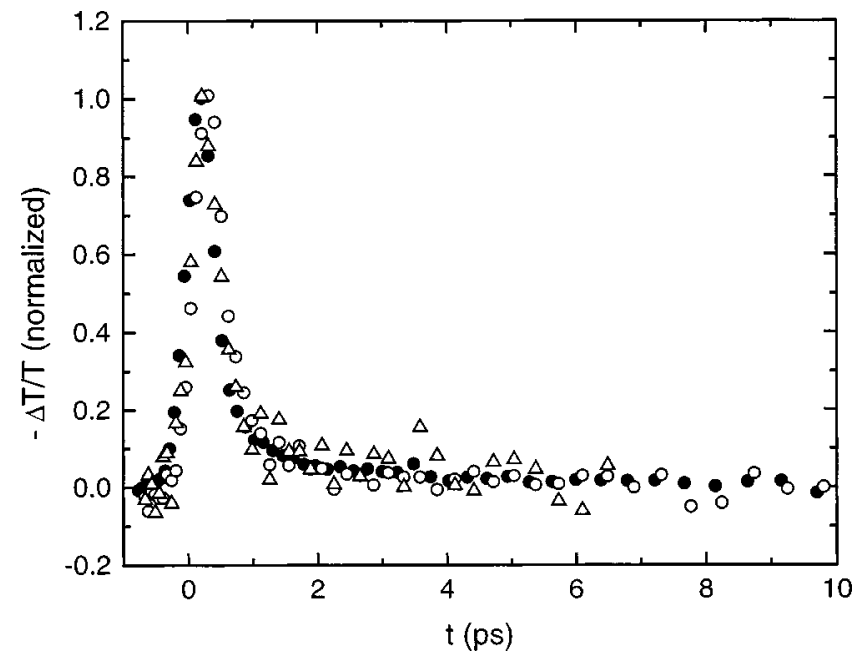

FIG. 3. Dynamics of the IRAV photoinduced absorption for different pump fluences: $285 \mu \mathrm{J} / \mathrm{cm}^{2}$ (filled circles), $136 \mu \mathrm{J} / \mathrm{cm}^{2}$ (open circles), and $60 \mu \mathrm{J} / \mathrm{cm}^{2}$ (open triangles). The pump photon energy was $1.87 \mathrm{eV}$ and the IR probe was at $1360 \mathrm{~cm}^{-1}$. Both polarizations were linear and parallel to the stretch direction. The curves were normalized for comparison of their dynamics.

dition, excitation at high photon energies is expected to increase the probability of generating charges on different chains (polarons), which are known to have a slower recombination dynamics. ${ }^{8}$

The above comparison has to be done with care. The charged soliton dynamics could depend on the excitation density due to bimolecular recombination, as we have observed previously for PPV derivatives. ${ }^{16}$ To investigate that possibility, we performed measurements with different pump fluences. The values of $-\Delta T / T(0)$ extracted from curve fitting were linearly proportional to the pump fluence, as expected for direct photogeneration of charged solitons. Furthermore, both the maximum of $-\Delta T / T(t)$ and the values of $-\Delta T / T(0)$ were linearly related, indicating that bimolecular recombination is not significant. Figure 3 also displays three normalized measurements of $-\Delta T / T(t)$ at different pump fluences, showing that the recombination dynamics is independent of excitation density, at least for the density range of our measurements.

The excitation spectrum, from 0.86 to $4.67 \mathrm{eV}$, for ultrafast charged soliton photogeneration is shown in Fig. 4, together with an absorption spectrum for transpolyacetylene. ${ }^{25}$ The results are plotted as twice the soliton pair photogeneration quantum efficiency times the cross section for the IRAV mode at $1370 \mathrm{~cm}^{-1}\left(2 \sigma \phi_{\mathrm{ch}}\right)$. This can be directly determined from $\Delta T / T(0)$ and the measured pump fluence at each excitation wavelength. The results were corrected for the pump transmission through the sample (detectable below $1.35 \mathrm{eV}$ ), but not for reflection or scattering losses. The most striking feature is the onset of charged soliton pair photogeneration at $\sim 1.0 \mathrm{eV}$. The quantum efficiency for charged excitation is nearly independent of photon energy over a wide range, except for a modest peak of $\phi_{c h}$ at $\sim 1.5 \mathrm{eV}$. The onset energy agrees well with that predicted for the formation energy of a soliton pair $2 E_{g} / \pi$ within the 


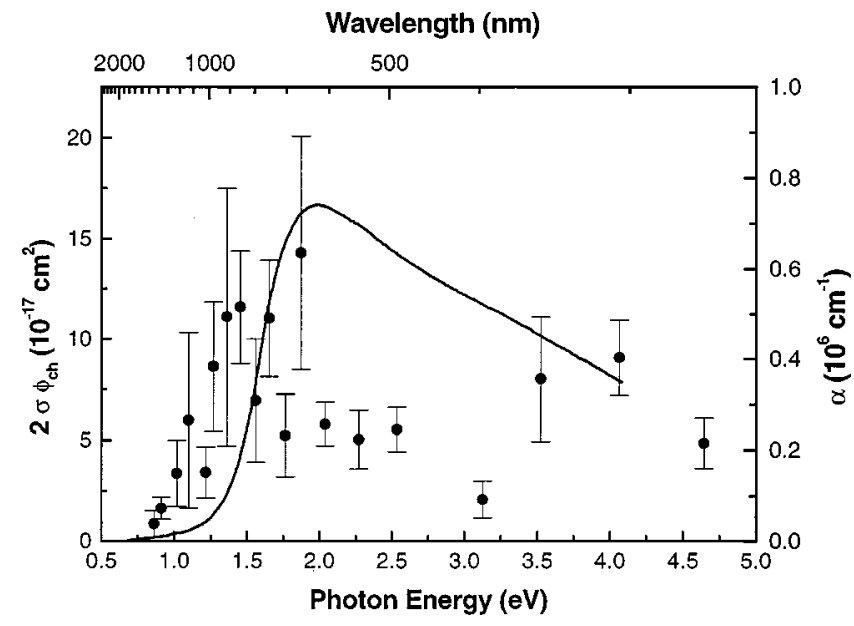

FIG. 4. Excitation spectrum for ultrafast charged soliton photogeneration in oriented trans-polyacetylene (filled circles). The IR probe was kept at $1360 \mathrm{~cm}^{-1}$. The solid line is the absorption spectrum taken from Ref. 25.

SSH model. ${ }^{3}$ In the literature, values for $E_{g}$ range from 1.4 to $1.8 \mathrm{eV}^{3,10,12,25}$ If we take $E_{g}=1.6 \mathrm{eV}$, the SSH threshold should be $1.02 \mathrm{eV}$, in excellent agreement with the observed onset $(\sim 1.0 \mathrm{eV})$. This is remarkable, considering the simplicity of the model. Of course, the uncertainty in $E_{g}$ would lead to SSH thresholds ranging from 0.89 to $1.15 \mathrm{eV}$, but these values are within our uncertainty in determining the threshold. As noted in the Introduction, previous steady-state measurements of the $\phi_{\text {ch }}$ excitation spectrum using PIA and photoconductivity ${ }^{13,14}$ obtained very different results, where $\phi_{\text {ch }}$ drops exponentially from $E_{g}$ to the SSH threshold.

We can estimate the absolute value of the soliton pair photogeneration efficiency $\left(\phi_{\mathrm{ch}}\right)$ if we know the cross section $(\sigma)$ for the IRAV mode at $1370 \mathrm{~cm}^{-1}$. The cross section can be estimated from doping-induced IRAV absorption $(\alpha)$ data, ${ }^{17}$ using $\alpha=\frac{1}{2} N \sigma$, where $N$ is the doping density and the factor $\frac{1}{2}$ arises because the measurement was done under unpolarized light (the IRAV absorption is polarized along the polymer chain). The measured absorption coefficient at 1370 $\mathrm{cm}^{-1}$ was $\alpha \approx 250 \mathrm{~cm}^{-1}$ for an oriented trans $-(\mathrm{CH})_{x}$ film doped with 0.03 mole $\% I_{3}^{-}\left(N \approx 6 \times 10^{18} \mathrm{~cm}^{-3}\right)$. Therefore, $\sigma \approx 8.3 \times 10^{-17} \mathrm{~cm}^{2}$. However, the effective IRAV cross section for our ultrafast measurements should be reduced by approximately a factor of two because of the large bandwidth of the IR pulses (see inset of Fig. 1), resulting in an effective value of $\sigma \approx 4.1 \times 10^{-17} \mathrm{~cm}^{2}$. Thus, $\phi_{\mathrm{ch}}=100 \%$ corresponds to an ordinate $\sim 8.3 \times 10^{-17} \mathrm{~cm}^{2}$ in Fig. 4. Considering the uncertainty in our measurements, we conclude that $\phi_{\text {ch }}$ ranges from 50 to $100 \%$ for photon energies above $1.0 \mathrm{eV}$. This is remarkable when compared to our ultrafast IRAV measurements in PPV derivatives, ${ }^{16}$ where we found $\phi_{\mathrm{ch}}$ $\sim 10 \%$.

The results presented in this section are in general agreement with theoretical models describing the photoexcitation and recombination processes in polyacetylene. We observe the onset for ultrafast charge photogeneration near $2 E_{g} / \pi$, implying that the detected charges are indeed soliton pairs. They are generated within our time resolution of $\sim 250 \mathrm{fs}$, in agreement with other measurements ${ }^{6,7,9}$ and with predictions of $\mathrm{Su}$ and Schrieffer. ${ }^{4}$ If charged soliton pair production is the dominant process following optical absorption, then $\phi_{\mathrm{ch}}$ should be $100 \%$ for any excitation wavelength, below or above the gap. This is what we observe in our measurements for energies above $1.0 \mathrm{eV}$; below that, residual absorption in the sample due to defects or impurities dominate, and $\phi_{\mathrm{ch}}$ begins to drop. The very fast charge recombination (poorly resolved by our measurements) could be explained as follows. The photogenerated charged solitons quickly relax to their lowest energy of $\sim 2 E_{g} / \pi$ for a charged pair by increasing the pair separation. ${ }^{11,5}$ For excitation near this threshold, the photogenerated soliton pair must be already well separated and the photogeneration process must be assisted by nonlinear quantum fluctuations of the lattice in the ground state. Once well separated, the charged and neutral soliton pairs are almost degenerate. This should greatly facilitate charge recombination into a pair of neutral solitons, ${ }^{26}$ which do not contribute to the IRAV intensity. (Presumably, the neutral soliton recombination dynamics has been observed by PIA at other probe energies. ${ }^{6,7}$ ) We note that this charge recombination mechanism seems to be very efficient as well, since for photon energies near or below $E_{g}$ the survival probability of charged solitons is very small (see Fig. 2), although pumping well above $E_{g}$ may lead to a somewhat higher survival probability. This is consistent with the low $\phi_{\mathrm{ch}}$ obtained in steady-state measurements. ${ }^{13,22}$ This fast and efficient nonradiative decay mechanism could also explain why trans-polyacetylene is nonluminescent.

\section{CONCLUSION}

In summary, we have used photoinduced IRAV modes to study the photogeneration and recombination dynamics of charged solitons in polyacetylene with $\sim 250$ fs time resolution. We observe that solitons are generated within our time resolution and have a very fast recombination dynamics (initial decay $<1 \mathrm{ps}$ ), which is moderately sensitive to the excitation photon energy. The quantum efficiency for charged soliton pair formation $\left(\phi_{\mathrm{ch}}\right)$ is inferred from our measurements to be close to unity. The excitation spectrum for $\phi_{\mathrm{ch}}$ has an onset at $\sim 1.0 \mathrm{eV}$ and a relatively weak photon energy dependence out to $4.7 \mathrm{eV}$. These results imply that photoexcitation of polyacetylene above the SSH threshold of $2 E_{g} / \pi$ leads to ultrafast charged soliton pair formation with nearly unity quantum efficiency, followed by very fast and almost complete recombination.

\section{ACKNOWLEDGMENTS}

This work was supported by the Air Force Office of Scientific Research under Grant No. F49620-99-1-0031 and the NSF under Grant No. DMR 00968202. Work in Korea was supported by the National Research Laboratory (NRL) program M1-0104-00-0023 of the Ministry of Science (MOST) Korea. 
*Present address: Departamento de Física, Faculdade de Ciências, Universidade Estadual Paulista, CP 473, Bauru-SP 17015-970, Brazil.

† Author to whom correspondence should be addressed. Email address: ajh@physics.ucsb.edu

\#Also at Physics Department and Materials Department, UCSB.

${ }^{1}$ C. K. Chiang, C. R. Fincher, Y. W. Park, A. J. Heeger, H. Shirakawa, E. J. Louis, S. Gau, and A. G. MacDiarmid, Phys. Rev. Lett. 39, 1098 (1977).

${ }^{2}$ M. D. McGehee, E. K. Miller, D. Moses, and A. J. Heeger, in Advances in Synthetic Metals, edited by P. Bernier (Elsevier, Lausanne, 1999), p. 98; A. J. Heeger, Angew. Chem. Int. Ed. Engl. 40, 2591 (2001).

${ }^{3}$ A. J. Heeger, S. Kivelson, J. R. Schrieffer, and W.-P. Su, Rev. Mod. Phys. 60, 781 (1988), and references therein.

${ }^{4}$ W.-P. Su and J. R. Schrieffer, Proc. Natl. Acad. Sci. U.S.A. 77, 5626 (1980).

${ }^{5}$ S. Kivelson and W.-K. Wu, Phys. Rev. B 34, 5423 (1986).

${ }^{6}$ C. V. Shank, R. Yen, J. Orenstein, and G. L. Baker, Phys. Rev. B 28, 6095 (1983).

${ }^{7}$ Z. V. Vardeny, J. Strait, D. Moses, T.-C. Chung, and A. J. Heeger, Phys. Rev. Lett. 49, 1657 (1982); C. V. Shank, R. Yen, R. L. Fork, J. Orenstein, and G. L. Baker, ibid. 49, 1660 (1982).

${ }^{8}$ M. Yoshizawa, T. Kobayashi, K. Akagi, and H. Shirakawa, Phys. Rev. B 37, 10301 (1988).

${ }^{9}$ L. J. Rothberg, T. M. Jedju, S. Etemad, and G. L. Baker, IEEE J. Quantum Electron. 24, 311 (1988); L. J. Rothberg, T. M. Jedju, S. Etemad, and G. L. Baker, Phys. Rev. Lett. 57, 3229 (1986).

${ }^{10}$ T. W. Hagler and A. J. Heeger, Prog. Theor. Phys. Suppl. 113, 65 (1993).

${ }^{11}$ Z.-B. Su and L. Yu, Phys. Rev. B 27, 5199 (1983); J. P. Sethna and S. Kivelson, ibid. 26, 3513 (1982).

${ }^{12}$ G. B. Blanchet, C. R. Fincher, and A. J. Heeger, Phys. Rev. Lett. 51, 2132 (1983).
${ }^{13}$ J. Orenstein, Z. Vardeny, G. L. Baker, G. Eagle, and S. Etemad, Phys. Rev. B 30, 786 (1984).

${ }^{14}$ L. Lauchlan, S. Etemad, T.-C. Chung, A. J. Heeger, and A. G. MacDiarmid, Phys. Rev. B 24, 3701 (1981).

${ }^{15}$ U. Mizrahi, I. Shtrichman, D. Gershoni, E. Ehrenfreund, and Z. V. Vardeny, Synth. Met. 102, 1182 (1998).

${ }^{16}$ D. Moses, A. Dogariu, and A. J. Heeger, Phys. Rev. B 61, 9373 (2000); P. B. Miranda, D. Moses, and A. J. Heeger, ibid. 64, R081201 (2001).

${ }^{17}$ C. R. Fincher, Jr., M. Ozaki, A. J. Heeger, and A. G. MacDiarmid, Phys. Rev. B 19, 4140 (1979).

${ }^{18}$ B. Horowitz, Solid State Commun. 41, 729 (1982).

${ }^{19}$ Z. G. Soos, G. W. Hayden, A. Girlando, and A. Painelli, J. Chem. Phys. 100, 7144 (1994).

${ }^{20}$ H. Shirakawa, Synth. Met. 28, D1 (1989).

${ }^{21}$ Y. W. Park, C. Park, Y. S. Lee, C. O. Yoon, H. Shirakawa, Y. Suezaki, and K. Akagi, Solid State Commun. 65, 147 (1988).

${ }^{22}$ Z. Vardeny, J. Orenstein, and G. L. Baker, Phys. Rev. Lett. 50, 2032 (1983).

${ }^{23}$ The ultrafast IRAV absorption we observe results from the lattice distortions induced by the presence of a charge in the polymer chain. These distortions eventually form a charged soliton in the relaxed (lowest energy) state. However, even if the lattice configuration has not attained its equilibrium configuration, IRAV modes will still be observed. Therefore, we are not necessarily probing the formation of a relaxed charged soliton, but rather the presence of charges in the chain (accompanied by some lattice distortion).

${ }^{24}$ E. J. Mele, Phys. Rev. B 26, 6901 (1982).

${ }^{25}$ G. Leising, Phys. Rev. B 38, 10313 (1988).

${ }^{26}$ Within the SSH model, the decay of a charged soliton pair into a neutral soliton pair is forbidden. However, it has been shown that even very small corrections to the model can have dramatic effects in breaking this selection rule for well separated soliton pairs (Refs. 5 and 11). 